\title{
CITY2NAVIGATION (C2N) - A NEW SERVICE FOR DIGITAL, FUTURE-PROOF URBAN TRAFFIC MANAGEMENT
}

\author{
Carsten Schürmann ${ }^{1, *}$, Dieter Geiger ${ }^{1}$, Marlene Picha ${ }^{2}$, Ralf Thomas ${ }^{2}$ \\ ${ }^{1}$ TCP International, Heilbronner Straße 150, 70191 Stuttgart, Germany - (cs, dg)@ @cp-international.de \\ ${ }^{2}$ Landeshauptstadt Stuttgart, Integrated Traffic Control Center, Mercedesstraße 33, 70372 Stuttgart, Germany - (marlene.picha, \\ ralf.thomas)@stuttgart.de
}

KEY WORDS: Traffic management, traffic management strategies, private routing services, mobility data marketplace, mobility services, ITS, data exchange, traffic control centres.

\begin{abstract}
:
Digital traffic management solutions are essential for the effective management of the continuing growth of road traffic. However, technical possibilities for implementing traffic management strategies by Traffic Control Centres are limited to a strategic network. At the same time, modern mobility apps from routing services offer road users many options to get informed and guided. The latter usually work independently from traffic management strategies of public authorities. Thus, different route recommendations not only lead to uncertainty of road users, but ultimately to a reduction in the effectiveness of the public traffic management strategies. A direct information exchange between both systems does not yet exist. This however would be a key to more efficient traffic management. City2Navigation therefore developed a technical concept for a nationwide implementation of a digital data exchange service $(\mathrm{C} 2 \mathrm{~N}$ service) to link public traffic management with routing services of private vendors. This service fills the gap between both group of actors, thereby serving as a crucial building block for digital traffic management in response to the goals of European and national frameworks for Intelligent Transport Systems (ITS).

The $\mathrm{C} 2 \mathrm{~N}$ service not only promotes the cooperation of public authorities with private routing services, it also offers a variety of opportunities to develop new business models. It is a complement to $\mathrm{C} 2 \mathrm{C}$ and $\mathrm{C} 2 \mathrm{X}$ communication solutions in road transport, ultimately also enabling future possibilities for municipalities to conduct efficient and sustainable traffic management.
\end{abstract}

\section{INTRODUCTION}

\subsection{Starting point: A lack of cooperation}

The continuing increase in motorised individual and road freight transport, coupled with a growing awareness of its negative environmental and climate impacts, are challenging public traffic management.

The possibilities for implementing urban traffic management strategies (TMS) by traffic control centres (TCC) are currently technically limited: For example, not all traffic lights can be controlled remotely, dynamic signposts and other information systems (e.g. parking guidance systems) are only available along selected strategic roads and at selected points in the centres of major cities, and also the possibilities to publish information as TMC messages are limited - both in terms of scope and quality of their information content (ISO, 2013). In general, only larger cities have a TCC, so that small and medium-sized towns currently have only little opportunities to take active traffic management measures.

In addition, further demands are being imposed on urban traffic management, such as ensuring urban and environmentally friendly routing including intermodal traffic management, the optimisation of cargo and delivery traffic and, in the future, the management of autonomous vehicles. Politicians and society would like to see corresponding traffic control measures in the future along roads without roadside actuators, also in smaller and

\footnotetext{
* Corresponding author

1 In this paper, we define public authorities as all public institutions in large, medium-sized, and small cities, as well
}

medium-sized towns, and a much more targeted and precise control of individual traffic or specific vehicle types.

At the same time, road users enjoy a wide range of technical options to get informed and guided through traffic, to find the cheapest means of transport and the best traffic routes, depending on the purpose and destination of their trips. Modern, convenient, and intermodal mobility apps offered by private routing services are playing an increasingly important role in this.

However, the latter have so far acted on a large scale independently and uncoordinated from the interests and traffic management strategies of public authorities ${ }^{1}$. While routing services design their services to optimize routes for an individual user, public traffic management strategies are focusing towards optimising the overall transport system, which in many cases leads to conflicts with private routing services and thus ultimately to uncertainty by car drivers when they receive divergent route recommendations (Figure 1).
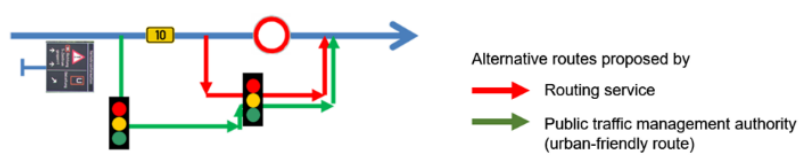

Figure 1. Divergent routes proposed by routing service and public traffic management authority.

Even worse, in individual cases, dangerous situations or even accidents can occur if a driver spontaneously changes his mind

as higher-level state or federal authorities that are responsible for the management and control of road traffic and public transport. 
before a junction and then follows the public signs instead of the recommendations of his routing service (or vice versa).

A direct information exchange between public systems and those of routing services, which could help preventing such conflicts, and could support a close coordination between both, does not exist until now, or only in pilot studies in form of siloed solutions.

Ultimately, the lack of cooperation leads to a non-exploitation of traffic management potentials and lacking effectiveness of public TMSs, along with a loss of user confidence in both public sector strategies and routing service recommendations.

If municipalities, including small and medium-sized ones without traffic control centres, would be enabled to publish their traffic management strategies in a standardised digital form (spatial, temporal, intermodal, vehicle and trip purpose-specific), and the routing services would take up this information cooperatively, mutual synergy effects would be created contributing to increased efficiency in traffic management and thus provide better results for the individual road user. As a side effect, the route optimization for the individual user would then also move closer to the optimum of the overall traffic system, thereby increasing the efficiency of the entire transport system.

To facilitate such a cooperation, some technical requirements are needed which to date are not yet available in a wider scope:

- Public authorities, including those without TCC, should be enabled to digitally define and publish TMS. Suitable and easy-to-use software tools for managing and publishing TMS are not yet available.

- All TMS need to be published via a central information broker from which the TMS can be retrieved by routing services. Solutions to directly connect individual public authorities with individual routing services bilaterally are probably not realistic given the large number of actors at both sides and the effort to create bilateral exchange relationships.

- The handling of the data broker must be made as simple as possible to facilitate the work of inexperienced users.

- The TMS messages must be published in a standardized digital format, allowing for an automated retrieval and processing through routing services.

\subsection{Legal frameworks and previous works}

The European legal framework for this initiative is set by the ITS Directive (European Parliament and the Council, 2010) and corresponding specifications, called Delegated Regulations (European Commission, 2017). According to these regulations, actors in the transportation system are obliged to provide and exchange traffic-related data via specified mechanisms. Part of such mechanisms are National Access Points (NAPs), being digital interfaces for ITS data, to be implemented in each EU member state (Jorna et al., 2018; Kleine et al., 2018).

The major motivation behind this framework is to make ITS more efficient and harmonised across Europe. The ITS Directive has been implemented by national laws in EU member states. Corresponding national activities are often concretised in national action plans, e.g. in Germany as the ITS Action Plan "Road" by the Federal Ministry of Transport and Digital Infrastructure (BMVI, 2017).

Data exchange in this context also covers traffic management measures and TMS published by public authorities, which are supposed to be re-used by information service providers, such as routing or mobility services. So far, however, no such data exchange has been established on a wider scale, i.e. across geographical and administrative levels. This is especially the case in Germany with a rather decentralised landscape of road authorities. One reason for the lacking data provision are, among others, the varying digitalisation levels of road authorities as potential data providers, and a lacking harmonisation of corresponding ITS data ecosystems.

For the harmonisation of such data ecosystems, so-called ITS reference architectures have been developed for certain domains, for example for multimodal traveller information (MRK, 2018). City2Navigation adds another ITS reference architecture to these, namely for TMS exchange between road authorities and routing services.

Some pilot projects have already been initiated across Europe, proving the viability of cooperation concepts between road authorities and routing services, and showcasing the technical feasibility of a $\mathrm{C} 2 \mathrm{~N}$ service with advanced routing services with the integration of TMS. In Germany, the NAVIGAR and LENA4ITS projects (Sabow et al., 2016; Von der Ruhren et al., 2014) must be mentioned, at European scale SOCRATES ${ }^{2.0}$ (SOCRATES 2.0 partners, 2019) for important previous works.

The potential of a $\mathrm{C} 2 \mathrm{~N}$ service was tested in NAVIGAR in a demonstration phase (June to December 2015) with 300 voluntary test riders in the Greater Stuttgart region. This test operation was scientifically evaluated (Sabow et al., 2016, 20ff). Test drivers were equipped with a special version of a common mobile routing app that was enabled to took TMS messages from the City of Stuttgart into account in the individual routing. The test results were encouraging. During the test phase, the service functioned technically flawlessly. The test persons saw a great need for such a service and stressed that it definitively had to consider all available up-to-date information including TMS published by public authorities. According to the volunteers, in case of alternative route recommendations, the reason for such re-routing should be communicated to the end users.

However, these pilot projects were limited in terms of their geographical and temporal scope, but in doing so they can be considered as proofs-of-concept. City2Navigation connects to them but follows another approach: It aimed to develop a baseline architecture and implementation concept for a large-scale rollout, whereas the deployment will be realised hereafter in individual implementation projects. In other words, City2Navigations aimed at a standardization of the $\mathrm{C} 2 \mathrm{~N}$ service.

\subsection{Objective of City2Navigation}

The objective of the City2Navigation project was therefore to develop a concept and an ITS reference architecture for the introduction of a cross-organizational, German-wide $\mathrm{C} 2 \mathrm{~N}$ service and thus to create the technical basis for linking public traffic management to routing services; the latter one being an essential objective of the ITS Action Plan "Road" of the German Federal Ministry of Transport and Digital Infrastructure (BMVI, 2017). This makes the $\mathrm{C} 2 \mathrm{~N}$ service a crucial building block for future-oriented digital traffic management. The reference architecture is primarily dedicated to strategic aspects, the identification of roles and their responsibilities, as well as value creation and governance of the service. In this way, it makes a significant contribution to the consistent definition of the core building blocks of the $\mathrm{C} 2 \mathrm{~N}$ service. In addition to the reference architecture, the project developed a requirements specification for the so-called "strategy editor" (product requirements 
document), a cooperation and evaluation concept, and identified framework conditions and further requirements for its implementation. With these objectives, the City2Navigation project contributes to the standardization of the $\mathrm{C} 2 \mathrm{~N}$ service in Germany.

\section{THE C2N SERVICE}

\subsection{Technical components}

Six key technological components make up the $\mathrm{C} 2 \mathrm{~N}$ service (Figure 2):

The strategy editor (1) is the central access point for public authorities to the $\mathrm{C} 2 \mathrm{~N}$ service. It is used for the definition, editing, administration, publication, activation, termination, and evaluation of TMS. In large cities the editor is supposed to be connected to existing traffic management systems. When a TMS is activated, it is automatically published by the strategy editor via the Mobility Data Marketplace (MDM) ${ }^{2}$ (so-called "forward channel").

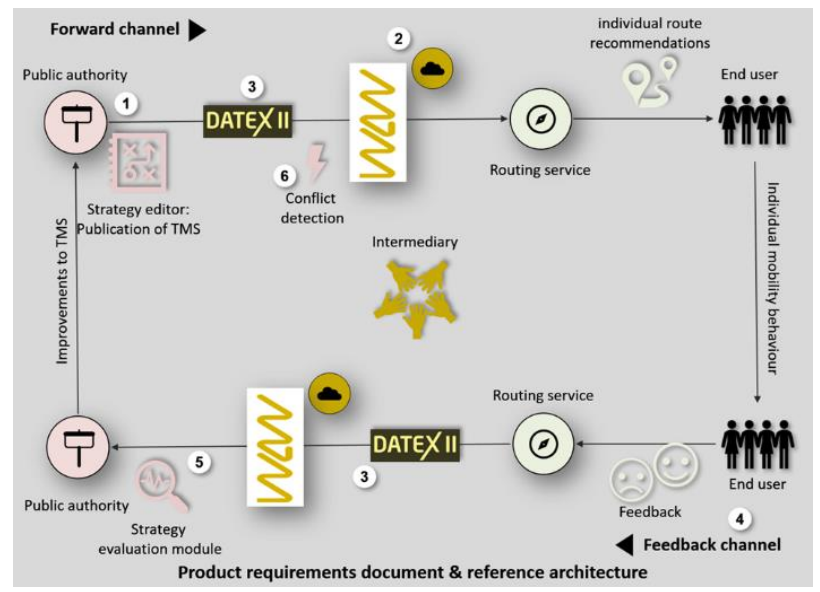

Figure 2. Interaction of the components of the $\mathrm{C} 2 \mathrm{~N}$ service.

The MDM (2) is the central information hub of the $\mathrm{C} 2 \mathrm{~N}$ service. Through this broker, the active TMS of all participating municipalities are provided, from where they can be accessed by the routing services. Depending on the triggering event, a TMS publication may consist of one or more individual traffic management measures and actions, including potential changes to the "map" (for instance, changes to the maximum permitted speeds on a road section) or recommended alternative routes.

Information is passed on via the MDM in a standardized manner via defined DATEX II profiles (3), which allow immediate and automated information processing by the routing services.

For municipalities to continuously evaluate and improve the quality and effectiveness of their TMS and of the TMS publications, the concept foresees a so-called "feedback channel" (4). Information about the degree of adoption of the TMS into routing recommendations should be sent from the routing services via the MDM to the publishing municipality in DATEX II format. This format will also be the technical basis for further

${ }^{2}$ The MDM represents the German NAP, operated by the Federal Highway Research Institute (BASt; MDM Website: www.mdm-portal.de/?lang=en).

${ }^{3}$ Plannable/schedulable events are events that occur at fixed dates or times (e.g. planned construction sites, festivities, and in-depth cooperation between public authorities and private routing services in the future, i.e., with regard to the joint identification of key performance indicators (KPIs) or the incentivization of traffic measures, and thus for the development of new business models.

The feedback channel information provided by the routing services is analysed and processed in the strategy evaluation module (5) of the strategy editor.

If two or more neighbouring municipalities activate certain TMSs at the same time, there may be cases where different TMSs negatively influence each other, resulting in conflicts in traffic management. This may especially be the case with ad hoc measures published in reaction to accidents or natural disasters. Therefore, as another core building block, the $\mathrm{C} 2 \mathrm{~N}$ service provides for automated conflict detection (6), identifying possible TMSs conflicts and informing the concerned public authorities.

\subsection{Optional extensions}

While basic functionalities of strategy evaluation and conflict detection are already provided by the strategy editor, the $\mathrm{C} 2 \mathrm{~N}$ service foresees options for offering advanced functionalities through specialized applications such as:

- semantic evaluation of the TMS for improved conflict detection,

- tools for (semi-automated) conflict resolution,

- mobile app of the strategy editor,

- detailed evaluation and simulation tool for strategy assessment, including traffic modelling approaches, provision of tools for analysing and evaluating user feedback, tools for implementation of KPIs.

In addition, the publication of TMS allows the development of further applications or new business models beyond routing applications, such as information systems, by private vendors.

\subsection{Use cases}

The $\mathrm{C} 2 \mathrm{~N}$ service can be used by public authorities in various use cases, for both schedulable events ${ }^{3}$ and in ad-hoc situations. Some of these use cases are traditional cases for traffic management (like TMS for traffic jams or construction sites, TMS for events or parking guidance), some of them are new use cases that are likely to emerge in the near future (Table 1) such as intermodal routing, TMS for large hubs, guidance for autonomous vehicles or geofencing applications.

The $\mathrm{C} 2 \mathrm{~N}$ service is aimed at approximately 10,000 municipalities and other public actors (counties, hub operators) in Germany that conduct traffic management. If one in three of these uses $\mathrm{C} 2 \mathrm{~N}$ and publishes on average five TMS per day, then approx. 15,000 TMS are processed daily via the $\mathrm{C} 2 \mathrm{~N}$ service. On the recipient side, these can be used by several dozen routing and mobility services. If only the German Federal Motorway Agency and all major cities with more than 100,000 inhabitants used C2N, approx. 50-70 percent of all road traffic in Germany would already be covered by the $\mathrm{C} 2 \mathrm{~N}$ service.

sporting events), or which recur regularly at certain times (e.g. morning rush hour). Traffic management can prepare for such events in advance and can develop appropriate strategies at an early stage. 


\begin{tabular}{|l|l|}
\hline Traditional use cases & Future use cases \\
\hline - Traffic jams & - Intermodal routing \\
- Road closures & - TMS for hubs (e.g. ports, \\
- Alternative routes & airports, freight villages) \\
- Construction sites & - Access restrictions for \\
- Event management & specific vehicles \\
- Parking guidance & - TM for MaaS \\
- TMS in case of accidents & - Kerbside management \\
or natural hazards & - Autonomous vehicles \\
- TMS to avoid traffic jams & - Geofencing applications \\
- Urban \& environmentally & - Incentivisation \\
friendly routing & - Key performance \\
- Priority routes (e.g. heavy & indicators (KPIs) \\
trucks, goods delivery) & - Cooperative traffic \\
& management \\
\hline
\end{tabular}

Table 1. Traditional and future use cases of $\mathrm{C} 2 \mathrm{~N}$ service.

The strategy editor is a map-based application for defining, storing, managing, and activating appropriate TMS for all such use cases.

\subsection{Publishing a traffic management strategy}

As soon as an event occurs, the operator can activate a TMS manually, or it is activated automatically when the corresponding triggers (roadside sensors) detect an event.

A TMS is defined as a predefined action plan for taking appropriate measures to improve traffic flows or to proactively prevent interferences or negative events in the traffic system.

Upon activation, the TMS will be published by the strategy editor to the MDM and optionally also as TMC message or via internet or other dedicated media.

Usually, each TMS consists of a collection of two or more measures, and each measure consists of one or more individual actions with different actuators. Examples of measures and actions are (but not limited to):

- changes to traffic lights signal programs,

- lane management (closure or opening of lanes, shoulder usage),

- changes of speed limits,

- road closures and access restrictions (for all vehicles, or only for specific ones),

- $\quad$ ramp metering

- changes in one-way street regulations,

- prioritisation of public transport (such as bus lanes),

- parking management (e.g. closure of or opening of additional parking space),

- vehicle occupancy regulations

- alternative routes and priority routes (for general traffic, or for specific vehicles such as trucks), or

- incentivation measures (e.g. free public transport towards city centre)

Apart from the measures and actions themselves, all of which need to have a spatial reference, each TMS message will also include information about the

- reasons for its activation which is an important information for the routing service and for the end user to understand the rationale for a measure, bindingness (is the TMS message a pre-announcement, a recommendation or does it have a sovereign character) and prioritisation (vis-à-vis other TMS),

- period of validity / activation / duration, and

- target groups (modes, vehicle types, trip purposes, origin/destination points).

Measures can be valid for all road users, or it can be specified that they only apply to certain vehicle types (trucks, cars, diesel vehicles, etc.), trip purposes, traffic modes, or only for traffic with certain destinations or starting points. With the help of the $\mathrm{C} 2 \mathrm{~N}$ service, these specifications can be defined more targeted, precisely, and flexibly than current traffic management systems allow with their roadside traffic signs. All specifications may be assigned to the entire TMS, or only to individual measures or actions within a TMS.

Another extension of the possibilities for public authorities generated through the $\mathrm{C} 2 \mathrm{~N}$ service is its flexible time management. With the $\mathrm{C} 2 \mathrm{~N}$ service, TMS can either be activated immediately, or strategies can be published in advance (advance information), but only activated later. An example of this is event management: If a TMS for a sports event is to be activated on Saturday at 1 p.m., a pre-announcement can be published early on Saturday morning or even the day before informing routing services that the strategy will be active from 1 p.m. onwards. The routing services can then use this advance information to offer their customers better predictive routing. The flexible time management also allows to indicate the activation period (i.e. duration) or the end time of a TMS in the strategy message.

Each TMS message will be published by the strategy editor as DATEX II profiles in XML connotation. City2Navigation used DATEX II Version 3.1 (Standard 16157 Part 8) as a starting point and developed several enhancements to this standard to be able to cover all the above requirements. For example, the ReroutingManagementEnhanced namespace was considered one of the fundamental DATEX II namespaces required for the C2N service (Figure 3). To implement the new enhancements, a new extended namespace ReroutingManagementEnhanced2 was developed in City2Navigation including all amendments to the DATEX II profile while maintaining the old namespace for reasons of compatibility.

\subsection{The MDM as data broker for TMS}

According to the European Commissions' Delegated Regulation, the TMS are to be provided free of charge and in a nondiscriminatory manner to routing and mobility services via the respective national NAP, i.e. via the MDM in case of Germany.

The data providers (i.e. public authorities) create a corresponding TMS publication on the MDM for this purpose (Figure 4). The MDM itself bundles the TMS publications of all cities into a single TMS publication by means of a so-called collector function. This single publication can then be accessed by interested data recipients (i.e. routing and mobility services), provided they have subscribed to the TMS publication (MDM, 2017). The MDM thus represents a one-stop shop for all TMS in Germany.

The advantage of this approach is that there is no need for bilateral subscriptions between individual data providers and data recipients, but that the link between the two is generated and managed centrally by the MDM. The MDM does not modify the

\footnotetext{
${ }^{4}$ MaaS $=$ Mobility-as-a-Service services
} 
TMS publications, but it buffers them so that routing services can retrieve the complete set of current TMS at any time.

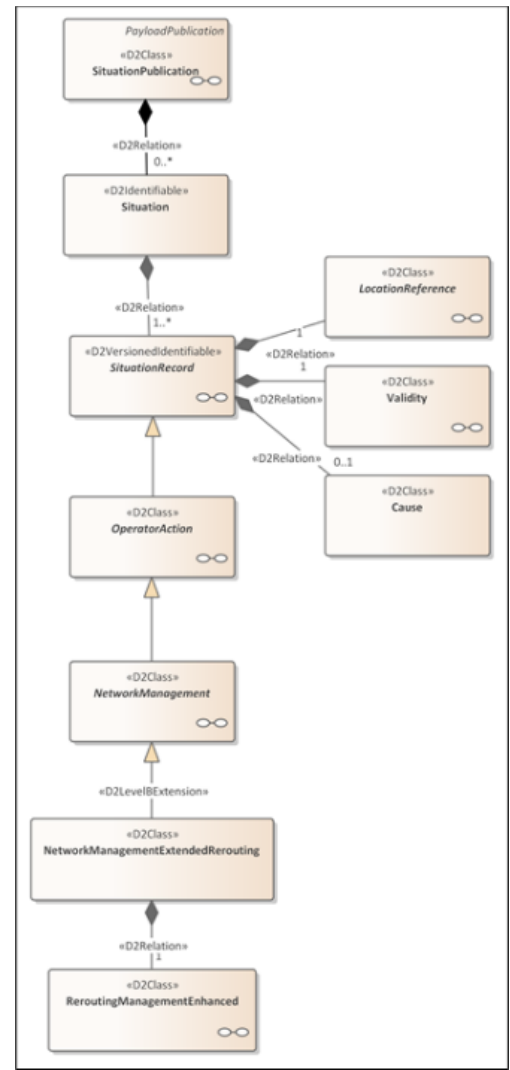

Figure 3. Embedding ReroutingManagementEnhanced into DATEX II situation model.

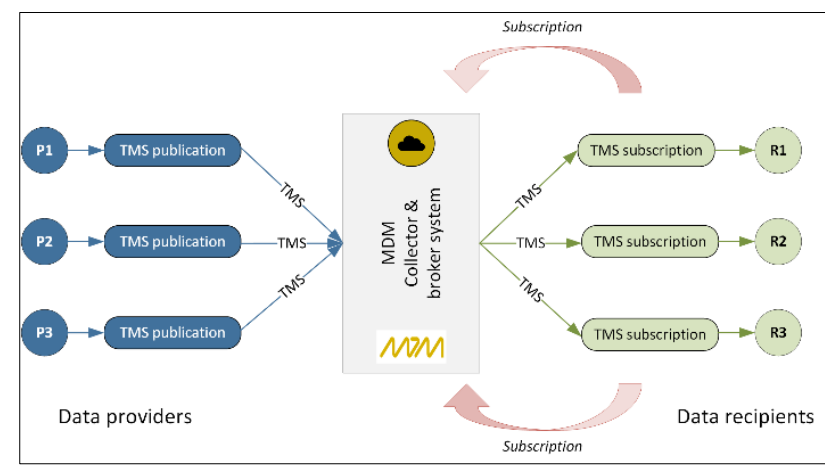

Figure 4. MDM as data broker.

The MDM itself has been operating successfully in Germany for several years and is used by many users to exchange data - even if often only bilaterally (BAST, 2020). Some central information services are already running via the MDM, for example the German-wide central information hub on petrol prices.

\section{BENEFITS AND NEW POSSIBILITIES}

Compared to the current situation, the $\mathrm{C} 2 \mathrm{~N}$ service expands the possibilities of traffic management for both public authorities and for private routing and mobility services in several aspects.

\subsection{Benefits for public authorities}

Public authorities responsible for traffic management benefit from the $\mathrm{C} 2 \mathrm{~N}$ service in different ways:

- They are enabled to carry out traffic management comfortably and comprehensively for streets and areas with and without roadside actuators (i.e. expanding the geographical scope beyond strategic networks), which will reduce costs for roadside infrastructures considerably.

- As a result, the service can also be used by public authorities that do not yet have TCC, traffic management systems and roadside actuators, like small and medium-sized towns.

- Traffic management measures can now be defined, published, and transmitted to the car drivers very specifically with respect to time, location, vehicle type and trip purpose, including intermodal measures.

- Strategies for congestion prevention can be proactively published, including advanced information on when a particular TMS will be activated and how long a TMS will stay active.

- Public authorities are alerted about possible conflicts of their TMS with those of other authorities.

- They can better analyse the impacts of their TMS, thereby improving the quality of their TMS gradually over time.

\subsection{Added value for private routing services}

There are also advantages for private routing services:

- They can use the more accurate TMS (including proactive ones) to generate even more individualized and target groupspecific route recommendations, thereby increasing customer satisfaction.

- They benefit from additional and better information, which is widely available.

- As the TMS are published in standardized DATEX II format, i.e. in machine-readable form, they can process the TMS automatically.

- They can retrieve all TMS of all authorities from one single data broker (i.e. the German NAP).

- Through the feedback channel, they get the opportunity to provide feedback to the authorities (including feedback from road users) and thus are enabled to support public authorities in developing even better traffic management measures in the future.

\section{IMPLEMENTATION}

\subsection{Guiding principles}

In addition to the presented technical solutions, the City2Navigation project also developed proposals for the implementation of the $\mathrm{C} 2 \mathrm{~N}$ service, based on the following guiding principles:

The $\mathrm{C} 2 \mathrm{~N}$ service should be introduced as quickly and seamlessly as possible in Germany. In this process, siloed solutions should be avoided, and different starting conditions and requirements of the municipalities should be considered. Temporary applications, for instance in form of pilot projects, should also be avoided in favour of permanent operational solutions.

\subsection{MDM and strategy editor}

In order to appeal to the largest possible number of users of the $\mathrm{C} 2 \mathrm{~N}$ service, the City2Navigation project provides on the one 
hand for a simplification in the handling of the MDM, in particular with regard to the registration, subscription, publication and pooling of TMS.

On the other hand, as stakeholder consultations have shown, different options for the implementation of the strategy editor are needed, accounting for the different requirements and starting conditions of public authorities:

- For large cities with a TCC, the extension of the existing TM editors would probably be the best and preferred option.

- For cities without a TCC but who use other related software (like construction site information systems), the extension of existing applications could be the preferred option.

- For medium-sized towns with own IT infrastructures, the development of stand-alone desktop software would probably be an option; and finally

- for small and medium-sized towns without large IT infrastructures, a centrally hosted web application would be the preferred solution.

\subsection{Role of the intermediary}

A central intermediary facilitating the $\mathrm{C} 2 \mathrm{~N}$ services and acting as a link between public authorities and routing services is needed. $\mathrm{He}$ represents the $\mathrm{C} 2 \mathrm{~N}$ service towards policy makers and the public, develops uniform terms of business, ensures its continuous technical operation, promotes the $\mathrm{C} 2 \mathrm{~N}$ service to the general public and implements the developed cooperation concept. The intermediary is supported by several decentralized mediators from the regions, establishing and maintaining close contacts with all regional and local actors involved.

The tasks assigned to the intermediary can be classified into technical, organisational, and content-related tasks (Figure 5). Technical tasks provide the technical foundations of the $\mathrm{C} 2 \mathrm{~N}$ services as well as parts of the permanent operations. Organisational and content-related tasks are partly also daily tasks but include also strategic tasks. The role of the intermediary can thus be further differentiated into a technical intermediary, operational intermediary, and strategical intermediary.

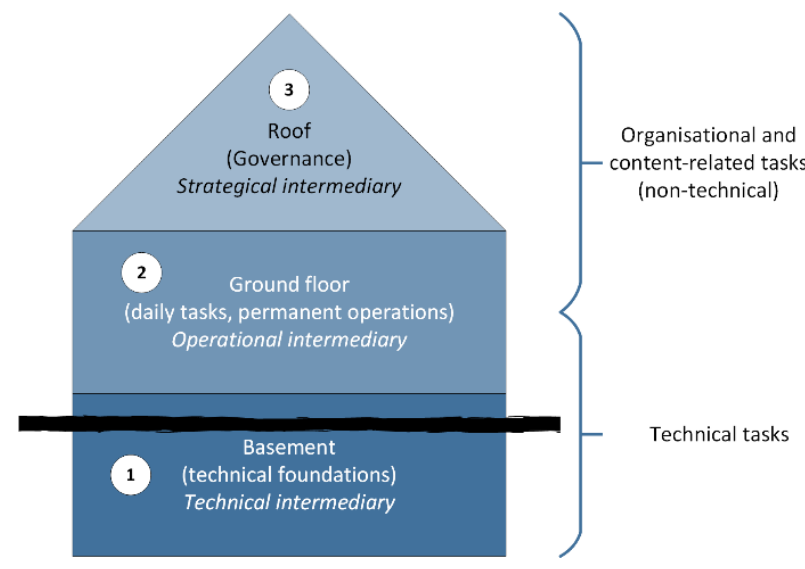

Figure 5. Intermediary: scope of work

\subsection{Implementation sequence}

In order to enable the $\mathrm{C} 2 \mathrm{~N}$ service addressing a maximum coverage of traffic in Germany from its very start of operation, selected large cities, the state authorities responsible for traffic management and the new Federal motorway authority should be among the first users of the $\mathrm{C} 2 \mathrm{~N}$ service in the first implementation stage. In a second stage, the $\mathrm{C} 2 \mathrm{~N}$ service should be extended to regional associations and organisations responsible for traffic management in an entire region on behalf of affiliated municipalities. In a third stage of expansion, the $\mathrm{C} 2 \mathrm{~N}$ service should then be extended to small and medium-sized towns.

Important steps have already been initiated to allow for a European-wide roll-out of the $\mathrm{C} 2 \mathrm{~N}$ service. The usage of standardized DATEX II profiles in C2N allow for easy transfer to other countries. The elaborated extensions of these profiles will be introduced into the international standardisation process for DATEX II. Thanks to the existing relevant European regulations, NAP exist in all EU Member States which should act as the national information broker - like MDM's role in Germany. Finally, the City2Navigation project already established active cooperation with related European projects such as SOCRATES 2.0 as a prerequisite for a future expansion of the $\mathrm{C} 2 \mathrm{~N}$ service towards entire Europe.

\subsection{Supporting materials and toolboxes}

To support implementation projects, the City2Navigation project developed various supporting materials and toolboxes:

1. Product requirements document for the strategy editor defining its mandatory and optional functionalities.

2. ITS reference architecture: Comprehensive documentation of all architectural modules, roles and actors including the implementation and further development of the DATEX II profiles.

3. Simplified access to the MDM: Suggestions for simplifying the processes of registration, subscription, publication, and pooling of TMS at the MDM.

4. Checklists: Separate checklists ("to-do" lists) for public authorities and routing services wishing to participate in the C2N service.

5. Certification: Proposals for the certification of implementations of the $\mathrm{C} 2 \mathrm{~N}$ service by the intermediary.

6. Evaluations and success factors: Suggestions on how public authorities can regularly evaluate the success of the $\mathrm{C} 2 \mathrm{~N}$ service in practice.

7. Documentation: All results of the City2Navigation project are published in a comprehensive final report on the website of the German Federal Highway Research Institute (BASt) in spring 2021.

These results provide a comprehensive toolbox for implementation projects of the $\mathrm{C} 2 \mathrm{~N}$ service and for interested stakeholders.

\subsection{Innovations}

The $\mathrm{C} 2 \mathrm{~N}$ service generates several innovations. The biggest one is the linkage of public traffic management to private routing services via the MDM as a central exchange platform. In addition, several other innovations were developed:

- The strategy editor allows easy editing, management, activation, and publication of the TMS.

- The TMS can be defined in higher detail (spatial, temporal, content-related) and thus more targeted than ever before.

Strategies can be published across all areas, even outside the strategic road network and in ad hoc situations, providing great flexibility for public authorities.

- Some DATEX II profiles have been extended to cope with these enhancements. 
- The private routing services have the possibility to give feedback to the public authorities on the quality of their TMS.

- Conflict detection and the evaluation module enable public authorities to continuously evaluate and improve their strategies.

- The closed information loop enables new business models at different stages and is the technical prerequisite for the incentivisation of traffic management.

\section{THE FUTURE OF PUPBLIC TRAFFIC MANAGEMENT}

\subsection{Next steps}

Now that the framework for the $\mathrm{C} 2 \mathrm{~N}$ service exists, the task is to realise it in implementation projects. Clear statements by the German government and the European Commission to promote such digital solutions already exist. To make the $\mathrm{C} 2 \mathrm{~N}$ service attractive for routing and mobility services,

(a) as many public authorities as possible must be convinced as quickly as possible to use the $\mathrm{C} 2 \mathrm{~N}$ service, first and foremost the newly founded Federal Motorway Authority in Germany.

(b) In addition, the intermediary as representative of the $\mathrm{C} 2 \mathrm{~N}$ service must be designated as soon as possible.

(c) Furthermore, it must be clearly communicated by the government that digital traffic management is a key element for the digitalisation of road transport.

(d) Although a nationwide roll-out of the $\mathrm{C} 2 \mathrm{~N}$ service is already a major challenge, ideally its basic ideas and technical principles should be spread throughout Europe. The extended DATEX II profiles will be forwarded to the corresponding European bodies for standardisation.

\subsection{Paving the way for future digital traffic management}

As illustrated, the overall concept of the $\mathrm{C} 2 \mathrm{~N}$ service is based exclusively on digital components and is thus a first step towards a fully digital traffic management, as outlined in the ITS Action Plan in Germany. Although in the immediate future all traffic management strategies will still be accompanied by corresponding roadside information (e.g. dynamic traffic signs), it is conceivable that medium to long term roadside signage will be completely dispensed as the $\mathrm{C} 2 \mathrm{~N}$ service has become established and digital information flows has become the new norm in road transport ${ }^{5}$. In view of the further digitalisation in the transport sector (autonomous vehicles, MaaS, etc.), the parallel digitalisation of traffic management is, from the point of view of city administrations, an absolute necessity to maintain scope for public authorities to conduct traffic management in the future. Digital traffic management solutions like the $\mathrm{C} 2 \mathrm{~N}$ services are also a mandatory prerequisite for the introduction of autonomous driving vehicles in cities.

So far, there have been a few pilot projects in Germany and Europe on which the City2Navigation project has built, which have tested the technical feasibility of the concept within geographically and temporally limited frameworks. These pilots have shown that in principle the technology is available and ready for implementation. However, the City2Navigation project also revealed the need for some technical improvements (e.g. handling the MDM) and extensions (e.g. regarding DATEX II profiles), although these are no insurmountable obstacles.
To establish access to the $\mathrm{C} 2 \mathrm{~N}$ service for public authorities, the strategy editor is indispensable, as is the assignment of the intermediary as a key actor for the implementation, promotion, and day-to-day-operation of the service.

City2Navigation has developed - for the first time ever - an overarching concept for the implementation of a $\mathrm{C} 2 \mathrm{~N}$ service, which can now be used as a guideline by follow-up implementation projects.

Interviews during the City2Navigation project have shown that already today many public authorities wish to publish their TMS but fail in doing so because of a lack of technology. On the other hand, routing services would like to integrate public TMS into their routing, but since such information is not yet widely available, they are not yet taking steps forward into this direction. This vicious circle can only be broken if the public sector initiates corresponding implementation projects in the near future. Support from the Federal and state levels is needed in this. The interest of all actors involved in the $\mathrm{C} 2 \mathrm{~N}$ service is there, but there is a lack of impetus from outside.

Further dissemination of the $\mathrm{C} 2 \mathrm{~N}$ service in Europe could be supported by relevant city networks such as POLIS. Finally, the problems described at the beginning are not a purely German phenomenon but occur in all cities and agglomeration areas. A Europe-wide use of the $\mathrm{C} 2 \mathrm{~N}$ service therefore would not only supports many cities across the continent in their daily traffic management but would simultaneously also increases the attractiveness of the service and thus the willingness of private routing services to participate in it.

\section{ACKNOWLEDGEMENTS}

This paper is based on parts of the research project "City2Navigation - Linking public traffic management to private routing services" carried out at the request of the German Federal Ministry of Transport and Digital Infrastructure, represented by the German Federal Highway Research Institute (Bast), under the "Research Programme for Urban Transport (FoPS)", project no. FE 77.0511/2017.

\section{REFERENCES}

BAST - Bundesamt für Straßenwesen (2020): MDM Benutzerhandbuch. Version 2.8.0 - 27.8.2020. Bergisch Gladbach: BASt.

BMVI - Bundesministerium für Verkehr und digitale Infrastruktur (2017). Zweiter Fortschrittsbericht zum IVSAktionsplan 'Straße' für Deutschland, https://ec.europa.eu/transport/sites/transport/files/2018_de_its_p rogress_report_2017.pdf

European Commission (2017): Delegated Regulation (EU) 2017/1926 supplementing Directive 2010/40/EU of the European Parliament and of the Council with regard to the provision of $E U$-wide multimodal travel information services. Official Journal of the European Union. Brussels.

European Commission (2019). Intelligent transport systems Action Plan and Directive,

5 See progresses in car-to-car (C2C) and car-to-X (C2X) communication. 
https://ec.europa.eu/transport/themes/its/road/action_plan/nap_e

European Parliament and of the Council (2010): Directive 2010/40/EU on the framework for the deployment of Intelligent Transport Systems in the field of road transport and for interfaces with other modes of transport. Official Journal of the European Union. Brussels.

ISO - International Standardising Organisation (2013). Intelligent transport systems - Traffic and travel information messages via traffic message coding - Part 1: Coding protocol for Radio Data System - Traffic Message Channel (RDS-TMC) using ALERT-C. ISO 14819-1. Geneva: ISO.

Jorna, R., Hendriks, L., Barr, J., Lubrich, P. (2018). National Access Points: Challenges for Success. In: Proceedings of the 25th ITS World Congress, Copenhagen, Denmark, 17-21 September 2018

Kleine, C., Hilti, F., Lubrich, T., Hoffmann, T. (2018). National Access Points and Municipalities - Best Practices from the German National Access Point MDM. In: Proceedings of the 25th ITS World Congress, Copenhagen, Denmark, 17-21 September 2018

MDM - Mobilitäts Daten Marktplatz (2017). Technische Schnittstellenbeschreibung 2.8.9-15. September 2017. Bergisch Gladbach: BASt.

MRK Management Consultants GmbH (2018). IVSReferenzarchitektur für multimodale Reiseinformation. Research project FE 03.0532/2014/IRB, commissioned by Federal Highway Institute (BASt).

Sabow, G.;, Krehle, G., Geiger, D., Möller, Chr., Thomas, R., Mann, M., Reifert, D., Zwicky, A., Schürmann, C., Wacker, M. (2016): NAVIGAR - Vernetzung von kommunalem Verkehrsmanagement und Navigationslösungen privater Anbieter. Stuttgart: ITS Baden-Württemberg e.V.

SOCRATES 2.0 Partners (2019). Setting the stage for interactive traffic management, SOCRATES 2.0 Deliverable, https://socrates2.org/download_file/force/144/184.

Von der Ruhren, St.; Kirschfink, H.; Ansorge, J.; KarinaWedrich, T.; Schopf, J.; Sparmann, J.; Wöbbeking, B.; Kannenberg, O. (2014): Maßnahmen zur Gewährleistung der Interoperabilität zwischen öffentlichem Verkehrsmanagement und individuellen Navigationsdiensten. Abschlussbericht von LENA4ITS. Frankfurt. 